\title{
Solitary Infiltrating Meningioma of the Trochlear Nerve: Case Report
}

\author{
Anita P. Bhansali ${ }^{1}$ Melissa M. Stamates ${ }^{1}$ John M. Lee ${ }^{2}$ Ricky H. Wong ${ }^{2}$ \\ ${ }^{1}$ Section of Neurosurgery, University of Chicago, Chicago, Illinois, \\ United States \\ 2 Department of Pathology and Department of Neurosurgery, NorthShore \\ University Health System, Evanston, Illinois, United States

\begin{abstract}
Address for correspondence Ricky $\mathrm{H}$. Wong, Northshore University Health System, Department of Neurosurgery, 1000 Central Street, Suite 880, Evanston, IL, 60201, United States
\end{abstract} \\ (e-mail: rwong@northshore.org).
}

J Neurol Surg Rep 2018;79:e63-e64.

\begin{abstract}
Keywords

- trochlear nerve

- meningioma

- nerve infiltration

- neuropathology

We report the case of a solitary meningioma infiltrating the trochlear nerve, the first in a patient without a neurocutaneous disorder or cavernous sinus involvement. The patient presented with diplopia was found to have a focal enhancing mass encompassing the trochlear nerve. Following surgical resection, pathological examination showed meningioma infiltrating the nerve itself, demonstrated on the included pathology images. A review of the literature and discussion of meningiomas infiltrating cranial nerves are included.
\end{abstract}

\section{Introduction}

Tumors of the trochlear nerve are rare; even schwannomas, the most common pathology, comprise only $\sim 30$ cases in the literature. ${ }^{1,2}$ Other pathologies include meningioma especially of the cavernous sinus, ${ }^{3}$ hemangioblastoma, ${ }^{4}$ cavernous angioma, ${ }^{5}$ and neurofibroma. ${ }^{6}$ Here we present an isolated trochlear nerve meningioma demonstrating infiltration of the tumor into the nerve tissue, which represents the first case of its kind to be reported. A brief review of cases in which meningioma infiltrated cranial nerves and the implications for tumor grading are discussed.

\section{Case Report}

A 52-year-old male with no medical history presented with blurred and double vision. He first developed dizziness and blurred vision 10 years prior during a soccer game. From that point, he noticed mild double vision with physical exertion. As the diplopia progressed, he started wearing prism glasses 5 years ago but still had symptoms when fatigued. He underwent left inferior rectus recession surgery and had resolution for $\sim 1$ year, but his double vision eventually returned. At presentation, he wore nonprescription prism glasses that resolved the diplopia but required replacement with stronger prisms every year. On physical exam, he demonstrated a mild, intermittent head tilt to the right. No obvious restriction of his extraocular movements was noted, but the patient reported worsening diplopia on right inferior gaze. Contrast-enhanced magnetic resonance imaging scan of the brain showed a $4 \mathrm{~mm}$ enhancing mass along the left ventrolateral pons in the area of the trochlear nerve, extra-axial but not dural-based.

The differential diagnosis included schwannoma, which was considered the likelier pathology, or meningioma, and the options of observation versus surgical removal were discussed. The progression of his diplopia suggested ongoing nerve damage caused either by compression or infiltration of the trochlear nerve. The options for observation or surgical intervention were discussed, and the patient elected to have surgery for diagnosis as well as surgical cure. The patient underwent a preauricular middle fossa craniotomy for resection of the mass. The trochlear nerve appeared to insert directly into the tumor, clinically more suggestive of a schwannoma. The proximal trochlear nerve was then sharply divided and the tumor was removed.

Pathological study demonstrated a meningiothelial meningioma (WHO Grade I) with no atypical histological features (-Fig. 1). The tumor was focally positive for EMA, but negative for progesterone receptors. The overall $K_{\mathrm{i}}-67$ proliferation index was 3\% to $4 \%$. A neurofilament stain demonstrated invasion by the meningioma into a peripheral nerve segment ( - Fig. 2 ). received

April 16, 2017

accepted

November 30, 2017
DOI https://doi.org/

10.1055/s-0038-1661005. ISSN 2193-6358. (c) 2018 Georg Thieme Verlag KG
Stuttgart · New York

License terms

(®) $\Theta \circledast$ 


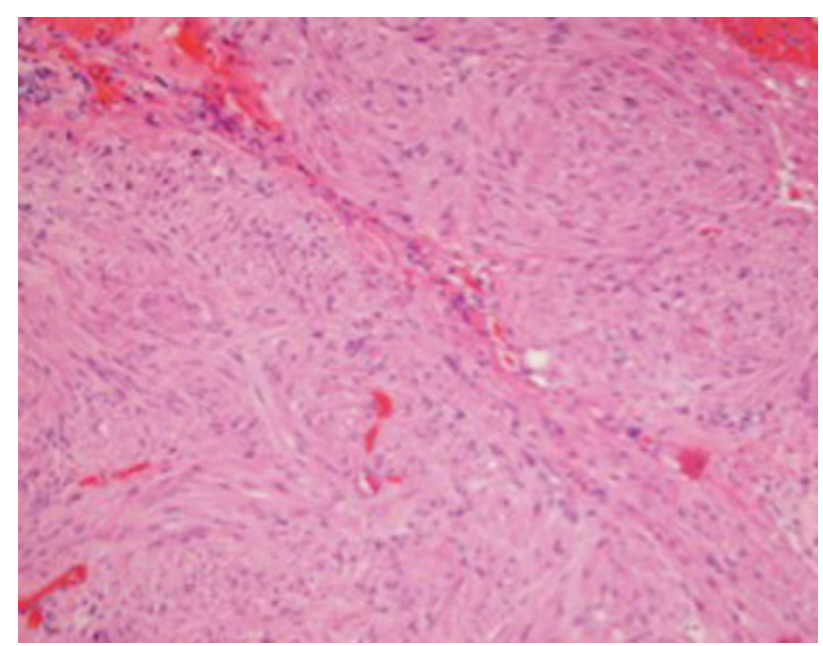

Fig. 1 Hematoxylin and eosin stain showing loose whorls and syncytia consistent with a meningothelial meningioma.

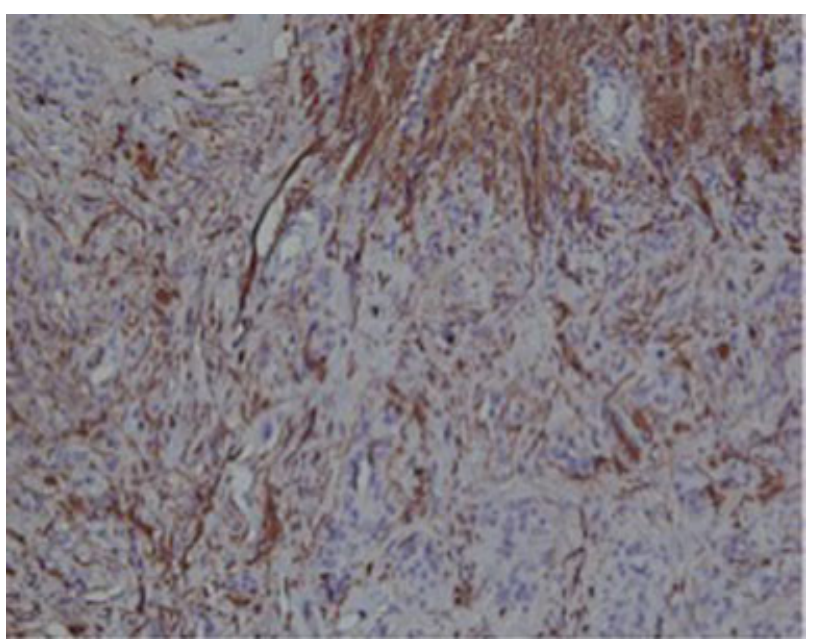

Fig. 2 Neurofilament immunostain showing invasion of tumor into peripheral nerve tissue.

\section{Discussion}

There are two prior cases in the literature of meningioma invading the trochlear nerve. The first case ${ }^{7}$ was described in a patient with von Recklinghausen's disease, who had fusiform mass of the right trochlear nerve found incidentally during resection of a right cerebellopontine angle. The mass was resected and pathological study demonstrated a meningothelial meningioma with nerve fibers found in the periphery. The second case $^{3}$ involved a cavernous sinus meningioma that had encased the right trochlear nerve. The nerve was resected, and pathological study demonstrated infiltration by a syncytial meningioma.

\section{Conclusion}

Nerve infiltration is not a criterion used in grading meningiomas, ${ }^{8}$ likely due to the rarity of cases and the lack of follow-up that would demonstrate that this feature is a risk factor for recurrence. Reports of nerve infiltration by meningioma include three cases of optic nerve invasion by optic nerve sheath tubular-diffuse type meningiomas; ${ }^{9}$ two cases of optic nerve invasion by intraorbital meningiomas, one transitional type found on autopsy and the other angiomatous type that was disease-free 28 years after resection; ${ }^{10}$ trigeminal (V3) nerve invasion by a syncytial meningioma; ${ }^{3}$ and accessory nerve invasion ${ }^{11}$ by a meningothelial type meningioma. While one case series of jugular foramen meningiomas reports that these tumors commonly invade the lower cranial nerves, ${ }^{12}$ there is limited evidence of this behavior to be found in the literature.

\section{References}

1 Boucher AB, Michael LM II. The middle fossa approach for the removal of a trochlear schwannoma. Case Rep Neurol Med 2014; 2014(14):672314

2 Younes WM, Hermann EJ, Krauss JK. Cisternal trochlear nerve schwannoma: improvement of diplopia after subtotal tumour excision. Br J Neurosurg 2012;26(01):107-109

3 Larson JJ, van Loveren HR, Balko MG, Tew JM Jr. Evidence of meningioma infiltration into cranial nerves: clinical implications for cavernous sinus meningiomas. J Neurosurg 1995;83(04):596-599

4 Tang Z, Wang C, Shi J. A solitary hemangioblastoma located on the trochlear nerve. J Clin Neurosci 2014;21(02):333-335

5 Manjila S, Moon K, Weiner MA, et al. Cavernous malformation of the trochlear nerve: case report and review of the literature on cranial nerve cavernomas. Neurosurgery 2011;69(01):E230-E238, discussion E238

6 Tripathy SR, Mishra SS, Deo RC, Mohanta I, Das D, Satapathy MC. Trochlear nerve neurofibroma in a clinically NF-1-negative patient; a case report and review of literature. World Neurosurg 2016;89:732.e13-732.e18

7 Tada T, Shigeta H, Kobayashi H, Kobayashi S, Sugita K. Trochlear nerve meningioma in von Recklinghausen's disease. Neurochirurgia (Stuttg) 1988;31(06):226-227

8 Louis DNOH, Wiestler OD. Cavenee WK (eds). WHO Classification of Tumours of the Central Nervous System. Lyon: IARC; 2007

9 Saeed P, Rootman J, Nugent RA, White VA, Mackenzie IR, Koornneef L. Optic nerve sheath meningiomas. Ophthalmology 2003; 110(10):2019-2030

10 Jain D, Ebrahimi KB, Miller NR, Eberhart CG. Intraorbital meningiomas: a pathologic review using current World Health Organization criteria. Arch Pathol Lab Med 2010;134(05):766-770

11 Tatagiba M, Koerbel A, Bornemann A, Freudenstein D. Meningioma of the accessory nerve extending from the jugular foramen into the parapharyngeal space. Acta Neurochir (Wien) 2005;147 (08):909-910

12 Sanna M, Bacciu A, Falcioni M, Taibah A, Piazza P. Surgical management of jugular foramen meningiomas: a series of 13 cases and review of the literature. Laryngoscope 2007;117(10):1710-1719 